\title{
Kinetic analysis of bacteriophage Sf6 binding to outer membrane protein a using whole virions
}

\author{
N. B. HUBBS ${ }^{1}$, M. M. WHISBY-PITTS², J. L. MCMURRY²
}

\begin{abstract}
${ }^{1}$ Department of Biology, Hanover College, 517 Ball Dr., Hanover, Indiana, 47243 USA; ${ }^{2}$ Department of Molecular and Cellular Biology, Kennesaw State University, 370 Paulding Ave., MD \#1201, Kennesaw, Georgia, 30144 USA
\end{abstract}

Received January 10, 2019; revised March 4, 2019; accepted October 16, 2019

\begin{abstract}
Summary. - For successful infection, viruses must recognize their respective host cells. A common mechanism of host recognition by viruses is to utilize a portion of the host cell as a receptor. Bacteriophage Sf6, which infects Shigella flexneri, uses lipopolysaccharide as a primary receptor and then requires interaction with a secondary receptor, a role that can be fulfilled by either outer membrane proteins (Omp) A or C. Our previous work showed that specific residues in the loops of OmpA mediate Sf6 infection. To better understand Sf6 interactions with OmpA loop variants, we determined the kinetics of these interactions through the use of biolayer interferometry, an optical biosensing technique that yields data similar to surface plasmon resonance. Here, we successfully tethered whole Sf6 virions, determined the binding constant of Sf6 to OmpA to be $36 \mathrm{nM}$. Additionally, we showed that Sf6 bound to five variant OmpAs and the resulting kinetic parameters varied only slightly. Based on these data, we propose a model in which Sf6: Omp receptor recognition is not solely based on kinetics, but likely also on the ability of an Omp to induce a conformational change that results in productive infection.
\end{abstract}

Keywords: Sf6; Shigella flexneri; OmpA; biolayer interferometry

\section{Introduction}

Virtually all viruses must translocate their genetic information into their respective host cells and replicate via the host cell machinery to produce progeny (Flint et al., 2000). dsDNA bacteriophages, which infect bacteria, are the most abundant viruses in the biosphere, with a global population estimated to be greater than $10^{30}$ (Hendrix, 2002). The molecular mechanisms that govern bacteriophage attachment to their hosts are not completely understood. Host recognition must be well coordinated by the virus in order to ensure fitness and progeny formation, as premature genome ejection does not result in a successful infection. One common mechanism bacteriophages employ is to utilize a portion of the host cell as a receptor (Poranen et al., 2002), and this can be through interactions with surface glycans, proteins, or both.

E-mail: hubbs@hanover.edu; phone: +1-812-866-7235. Abbreviations: $\mathrm{BLI}=$ biolayer interferometry; LPS = lipopolysaccharide; Omp = outer membrane protein
Teichoic acid, peptidoglycan, and other components of Gram-positive bacteria have been shown to be receptors for many phages (Chatterjee, 1969; Cleary et al., 1977; Wendlinger et al., 1996; Sao-Jose et al., 2006; Baptista et al., 2008; Rakhuba et al., 2010; Bertozzi Silva et al., 2016). For instance, \$29 recognizes glucosylated teichoic acid in Bacillus subtilis (Young, 1967). Bacteriophage SPP1, in addition to teichoic acid, also requires recognition of membrane protein YueB to irreversibly adsorb and commit to infection (Sao-Jose et al., 2006; Baptista et al., 2008). Lipopolysaccharide (LPS) and/ or proteins localized on the outer surface of Gram-negative bacteria are also used as phage receptors (Rakhuba et al., 2010; Bertozzi Silva et al., 2016). For instance, bacteriophage T7 recognizes the LPS of Escherichia coli (Gonzalez-Garcia et al., 2015) and phage S16 recognizes outer membrane protein C (OmpC) (Marti et al., 2013). Different forms of LPS are commonly used as phage receptors and attachment is regulated by either the length of LPS or by specific $\mathrm{O}$-antigen modifications, for phages such as P22 and Sf6 (Lindberg et al., 1978; Baxa et al., 1996). Sequence-diverse Omps are also commonly used as receptors by phages that infect Gram- 
negative hosts; examples include OmpA, OmpC, OmpF, LamB, FhuA, as well as others (Braun and Wolff, 1973; Randall-Hazelbauer and Schwartz, 1973; Hantke and Braun, 1978; Morona et al., 1985; Hashemolhosseini et al., 1994a,b; Smith et al., 2007).

Bacteriophage Sf6 is a short-tailed dsDNA virus that belongs to a subgroup of the family Podoviridae, the "P22like" phages (Morona et al., 1994). Sf6 infection of Shigella flexneri is a two-step process that utilizes both glycans and proteins during infection. First, Sf6 reversibly recognizes and then hydrolyzes LPS via its tailspikes (Freiberg et al., 2003; Porcek and Parent, 2015). Second, Sf6 interacts irreversibly with a protein receptor to commit to infection (Parent et al., 2014). Sf6 preferentially uses OmpA, but can also use OmpC when OmpA is absent (Parent et al., 2014). Sf6 can likely utilize a third, as of yet unidentified receptor, as infection still occurs in the absence of both OmpA and OmpC (Parent et al., 2014). Bacteriophage Sf6 has an inherent ability to utilize multiple Omps for infection (Parent et al., 2014). Although host range studies have generated mutants of other phages that can switch to utilize alternative receptors when under selection pressure (Morona and Henning, 1984; Drexler et al., 1989; Hashemolhosseini et al., 1994a,b), an innate ability to recognize multiple receptor types is not a common phenomenon, making Sf6 somewhat unique.

Our previous work showed that OmpA surface loops mediate Sf6 infection and confer host range (Porcek and Parent, 2015). Individual amino acid substitutions in OmpA loops result in a range of Sf6 infection efficiencies (Porcek and Parent, 2015). In an effort to better understand Sf6 interactions with OmpA, and how variations affect binding, we used biolayer interferometry (BLI) to determine the kinetics of these interactions. BLI is an optical biosensing technique used to measure the kinetic parameters of biomolecular interactions (Abdiche et al., 2008; Concepcion et al., 2009). It works by tethering one binding partner (the ligand, in this study, whole phage) to a fiber optic sensor tip. The ligand-loaded sensor is then dipped into a sample that contains a known concentration of the binding partner (the analyte, in this study, purified receptor proteins). White light reflects off of two optical layers in the tip, establishing an interference pattern, which is measured by a photodetector. Binding between ligand and analyte causes the distance between optical layers to increase, resulting in a shift in the interference pattern. The shift (in $\mathrm{nm}$ ) plotted against time in an association-then-dissociation experiment allows for determination of rate and affinity constants. Examination of several different analyte concentrations allows for robust global fits.

Previously published BLI kinetic analyses have used purified host receptors and studied interactions with purified viral receptor binding proteins (Xiong et al., 2013; Yang et al., 2015; Zhang et al., 2015), yet no such studies are published to date for bacteriophages. Here, we successfully immobilized intact Sf6 virions by amine crosslinking. To our knowledge, this also represents the first study of whole virion immobilization completed on the BLI platform. We determined the equilibrium dissociation constant of Sf6 to OmpA, and found it is $36 \mathrm{nM}$. Moreover, we showed that Sf6 bound to five variant OmpAs that demonstrated phenotypic changes (Porcek and Parent, 2015), yet the resulting kinetic parameters vary only slightly when compared to the native Shigella OmpA protein. These results suggest that the altered infection efficiencies observed in vivo are not solely dependent on the rate at which Sf6 interacts with OmpA.

\section{Materials and Methods}

Media and strains. Bacterial growth, plating experiments, and preparations of Sf6 phage stocks were all completed in Lysogeny broth (LB). Bacteriophage Sf6 (clear plaque mutant (Casjens et al., 2004)) was propagated on omp $A^{-} C^{-}$S. flexneri (dual ompA and $\operatorname{omp} C$ gene knock-out), as previously described (Parent et al., 2014). Phage used for in vitro genome ejection experiments were stored in phage buffer (10 mM Tris, pH 7.6 and $10 \mathrm{mM} \mathrm{MgCl}_{2}$ ) and phage used for BLI experiments were stored in $\mathrm{NaOAc}$ buffer (10 mM sodium acetate, $\mathrm{pH} 4.0$ and $2 \mathrm{mM} \mathrm{MgCl}_{2}$ ). S. flexneri strain PE577 was used for phage plating experiments (Morona et al., 1994). OmpA-TM proteins ("TM" = transmembrane portion of OmpA that lacks the periplasmic domain, and has been shown to be sufficient to induce genome ejection in vitro) were expressed in E. coli BL21/DE3/pLysS cells, unfolded in $6 \mathrm{M} \mathrm{GuHCl,} \mathrm{puri-}$ fied using Ni-NTA agarose matrix (Qiagen) in the presence of $6 \mathrm{M}$ urea, and then reconstituted by slow dialysis into $0.1 \%$ Triton X-100, as previously described (Parent et al., 2014; Porcek and Parent, 2015). The initial concentration of purified OmpA-TM was determined using a Bradford assay. We then created $0.2 \mathrm{mg} /$ $\mathrm{mL}$ stock solutions, ran a portion of each on a $15 \%$ SDS gel, and quantified the resulting bands by gel densitometry (BIORAD Gel Doc XR+) as previously described (Porcek and Parent, 2015) to ensure that the final concentration of variant OmpA-TM dilutions were identical to OmpA-TM $\mathrm{TS}_{\text {.flex }}$.

LPS extraction and in vitro genome ejections. Using a BulldogBio kit, S. flexneri LPS was extracted from PE577 as previously described (Parent et al., 2014). Sf6 was incubated at 25,30 , or $37^{\circ} \mathrm{C}$ with purified LPS $(0.5 \mathrm{mg} / \mathrm{ml})$ and OmpA-TM S.fex $(0.05 \mathrm{mg} / \mathrm{ml})$. The "percent remaining virions" is a measurement for the fraction of the population of phages that have not released their genomes after interaction with LPS and OmpA-TM and was calculated by dividing the plaque forming units (PFUs) in each reaction by the PFUs in buffer. To determine PFUs, plates were grown overnight at $30^{\circ} \mathrm{C}$.

Biolayer interferometry. Kinetic analyses of variant OmpA-TMs binding to Sf6 phage were performed on a FortéBio (Menlo Park, CA) Octet QK BLI instrument using amine reactive sensors (AR2G) 

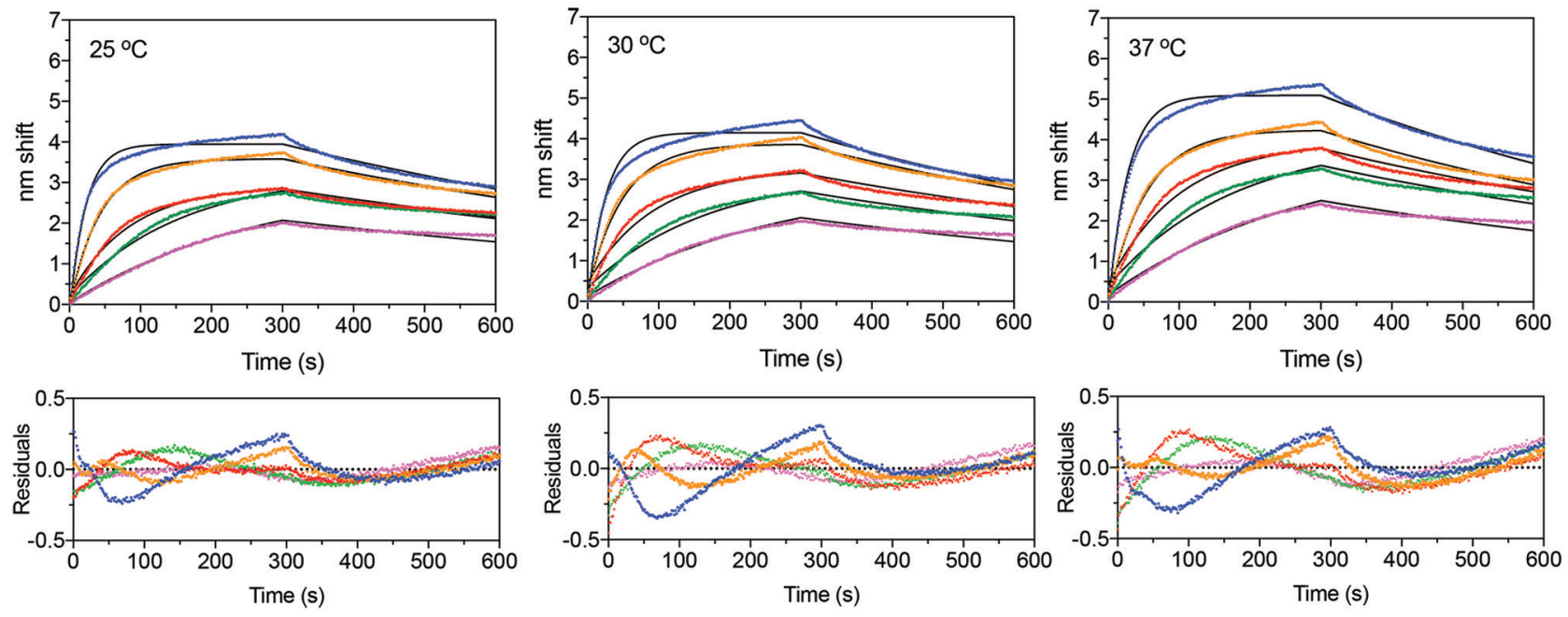

Fig. 1

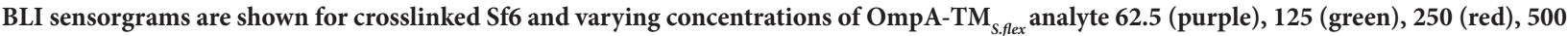
(orange), and 1,000 (blue) $\mathrm{nM}$ at 25,30 , and $37^{\circ} \mathrm{C}$

Reference subtracted raw data are shown as points and global 1:1 association-then-dissociation non-linear fits are shown as solid black lines. Association and dissociation times were $300 \mathrm{~s}$. Residuals are shown below the sensorgrams and are less than $10 \%$ of the total signal. Kinetic and equilibrium dissociation constants determined from the sensorgrams are shown in Table 1.

Table 1. Kinetic and equilibrium dissociation constants for Sf6 and OmpA-TM $_{\text {S.flex }}$

\begin{tabular}{ccccc}
\hline $\begin{array}{c}\text { Temperature } \\
\left({ }^{\circ} \mathbf{C}\right)\end{array}$ & $\boldsymbol{k}_{\text {on }}\left(\mathbf{M}^{-1} \mathbf{s}^{-1}\right)$ & $\boldsymbol{k}_{\text {off }}\left(\mathbf{s}^{-1}\right)$ & $\boldsymbol{K}_{\mathrm{D}}(\mathbf{n M})$ & $\begin{array}{c}\text { Saturation deter- } \\
\text { mined } \boldsymbol{K}_{\mathrm{D}}(\mathbf{n M})\end{array}$ \\
\hline 25 & $4.3 \times 10^{4}$ & $1.0 \times 10^{-3}$ & 23.3 & 82.3 \\
30 & $3.8 \times 10^{4}$ & $1.2 \times 10^{-3}$ & 31.2 & 99.6 \\
37 & $3.5 \times 10^{4}$ & $1.3 \times 10^{-3}$ & 36.4 & 89.9 \\
\hline
\end{tabular}

at 25,30 , or $37^{\circ} \mathrm{C}$. All final volumes were $200 \mu$ l. A stock of Sf6 phage in $10 \mathrm{mM}$ sodium acetate buffer, $\mathrm{pH} 4.0$ at a titer of $1 \times 10^{10}$ phage/ml was used to tether the phage to the sensor. The AR2G sensors were wetted and activated in $10 \mathrm{mM}$ sulfo-NHS (N-hydroxysulfosuccinimide) and $400 \mathrm{mM} \mathrm{EDC} \mathrm{(1-ethyl-3-(3} \mathrm{dimethyl-}$ aminopropyl)carbodiimide hydrochloride) for 300 seconds. Sensors were then dipped for 600 seconds in the phage stock to allow crosslinking, which was followed by quenching in $1 \mathrm{M}$ ethanolamine, $\mathrm{pH} 8.5$ for $300 \mathrm{~s}$. Baseline was established in $0.1 \%$ Triton X-100 (diluted in water) over a period of 300 seconds. Sensors were then exposed to various OmpA-TM analytes (ranging from $1,000 \mathrm{nM}$ to $7.8 \mathrm{nM}$ ) for $300 \mathrm{~s}$ to measure association. Dissociation was measured for $300 \mathrm{~s}$ by dipping the sensors into $0.1 \%$ Triton $\mathrm{X}-100$. Data were reference-subtracted using the signal from crosslinked phage exposed only to $0.1 \%$ Triton X-100. Nonspecific binding was measured by exposing a sensor without tethered phage to the highest concentration of OmpA-TM $\mathrm{TM}_{\text {sflex }}$ and was found to be negligible. Data were fit using GraphPad Prism 7 (GraphPad Software, La Jolla, CA, USA) and BiaEvaluation Soft- ware (GE Healthcare, USA). Experiments were performed in triplicate. Global fits were calculated from each set of experimental data, and overall there was relatively little binding variation between separate titrations.

\section{Results}

Temperature does not significantly change the kinetic parameters of Sf6 and OmpA binding

We purified the transmembrane domain of S. flexneri

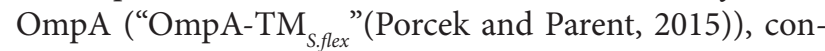
firmed we had functional OmpA-TMs by our previously reported assays (Parent et al., 2014; Porcek and Parent, 2015), and then measured the ability of OmpA-TMs to induce Sf6 genome ejection in vitro prior to performing BLI experiments. For all BLI experiments described herein, the ligand, Sf6, was immobilized on amine reactive (AR2G) sensors. OmpA-TMs reconstituted into detergent micelles were used as analytes. To ensure that sodium acetate, pH 4.0 buffer (a low pH buffer, in which phage are not typically stored, but which was necessary for tethering to sensors) had no effect on the phage, we monitored the titer of the phage stock over time, comparing it to phage stored in phage dilution buffer, $\mathrm{pH}$ 7.6, and found no significant differences. Moreover, we tested the ability of OmpA-TM ${ }_{\text {S.flex }}$ to induce genome ejection of Sf6 stored in $\mathrm{NaOAc}, \mathrm{pH} 4.0$ buffer and found it to be similar to previously published 
results (Parent et al., 2014; Porcek and Parent, 2015). In addition, Sf6 binding to OmpA-TM $\mathrm{T}_{\text {Sflex }}$ was specific, as phage Sf6 did not bind to other outer membrane proteins.

To determine kinetic parameters of Sf6 and OmpA$\mathrm{TM}_{\text {S.flex }}$ binding, we measured the change in interference patterns over time to generate sensorgrams at 25, 30, and $37^{\circ} \mathrm{C}$ (Fig. 1). The generated data were fit in GraphPad prism to a global 1:1 association-then-dissociation model (Fig. 1). Calculated kinetic parameters are shown in Table 1 . The analyte concentrations tested ranged from $62.5 \mathrm{nM}$ to $1,000 \mathrm{nM}$. Consistent with our hypothesis and published results from other bacteriophage and host receptor biosensing work (Bonaparte et al., 2005; Marti et al., 2013; Xiong et al., 2013; Wang et al., 2014; Yang et al., 2015; Zhang et al., 2015), OmpA-TM ${ }_{\text {S.flex }}$ bound Sf6 with nM affinity that varied only slightly with changes in temperature. Based on the calculated parameters OmpA-TM $\mathrm{T}_{\text {fflex }}$ bound Sf6 with relatively fast- on and slow-off kinetics. Overall, these data suggest that temperature differences do not significantly affect Sf6 binding to OmpA-TM ${ }_{\text {S.flex }}$.

\section{Sf6: OmpA-TM binding}

Observed binding was complex. As seen in Fig. 1, a 1:1 association-then-dissociation model (i.e. one-state fit) fits the data relatively well, though a secondary component is noticeable at higher concentrations. Data were collected from broad ranges of concentrations of OmpA-TM Sflex $_{\text {at }}$ at $37^{\circ} \mathrm{C}$ and fit to several other models including two-state parallel and conformational change models, for which the fits were ambiguous or poor (data not shown). Goodnessof-fit is described by $\chi^{2}$ (Yang et al., 2016); the lower the $\chi^{2}$, the better the model describes the fit of the data. In addition to $\chi^{2}$, the one-state model was the only one to pass the F test, a standard statistic for ensuring validity of a kinetic model (Burguillo et al., 1983). Additionally, equilibrium binding analyses of the data shown in Figure 1 yielded $\mathrm{K}_{\mathrm{D}} \mathrm{s}$ of 82.3, 99.6, and $89.9 \mathrm{nM}$ (Table 1), which are all essentially indistinguishable from each other and in reasonable proximity to the constants derived from the kinetic models, i.e. the secondary component (seen with all OmpA-TM variants) is minor and probably reflective of some artifact rather than biology, e.g. ligand presentation. Another potential explanation is that detergent micelle size varies within a preparation, yet is usually smaller than the membrane-inserted portion of a single OmpA $\beta$-barrel $(\sim 19 \mathrm{kDa})$ (Kleinschmidt et al., 1999). Therefore, in some cases OmpA stoichiometry within micelles may be a 1:1, but in other cases, multiple micelles likely aggregate around a single OmpA protein, as reported in (Kleinschmidt et al., 1999). This variance likely explains at least part of the complex behavior of our data at high analyte concentrations, and why the residuals are not random at these concentrations.

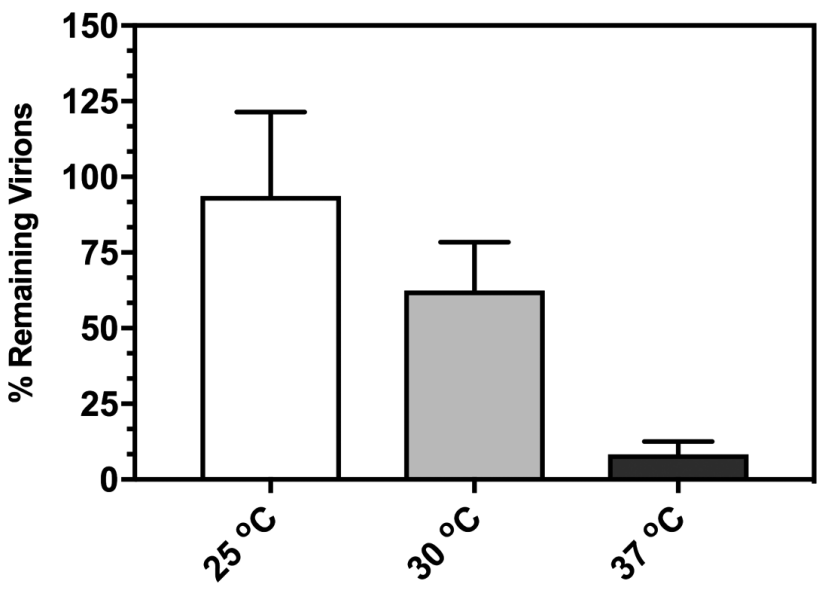

Fig. 2

Sf6 in vitro genome ejection efficiency increases with temperature Ejection efficiency of $S f 6$ incubated at 25,30 , or $37^{\circ} \mathrm{C}$ for 10 min with LPS +

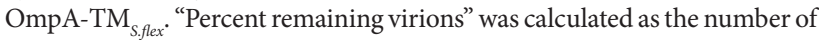
PFUs remaining after incubation at each temperature divided by the number of PFUs when treated with buffer only. Each data point is an average of at least three separate experiments; error bars signify one standard deviation.

\section{Sf6 genome ejection efficiency is highest at physiological temperatures}

We were surprised that the binding kinetics did not change greatly with temperature as phage ejection can often be affected by temperature (Andres et al., 2012; de Frutos et al., 2005; Killmann et al., 1995; Mackay and Bode, 1976; Sao-Jose et al., 2006). Therefore, we tested if Sf6 genome ejection was affected in vitro using our standard assay (Parent et al., 2014; Porcek and Parent, 2015) and measured the efficiency of genome ejection at 25,30 , and $37^{\circ} \mathrm{C}$ (Fig. 2). Reactions were incubated for $10 \mathrm{~min}$, which is within the timeframe of the lengths of BLI association phases. Consistent with previously reported data, at $37^{\circ} \mathrm{C}$ the majority (>95\%) of Sf6 virions have lost their genomes at $10 \mathrm{~min}$ post initiation of ejection (Parent et al., 2014; Porcek and Parent, 2015). However, as temperature decreased, the observed genome ejection efficiency in vitro also decreased. For example, at $30^{\circ} \mathrm{C}, \sim 40 \%$ of virions have lost their genomes and only $\sim 10 \%$ at $25^{\circ} \mathrm{C}$. Therefore, all subsequent BLI experiments performed were completed at $37^{\circ} \mathrm{C}$.

\section{Sf6 binds different OmpA-TMs at similar affinities}

Previously reported amino acid substitutions in OmpA resulted in altered infection efficiencies of Sf6 ejection in vivo and in vitro (Porcek and Parent, 2015), and it was thought that these changes may be due to differences in binding affinities. We purified various OmpA-TMs: one 

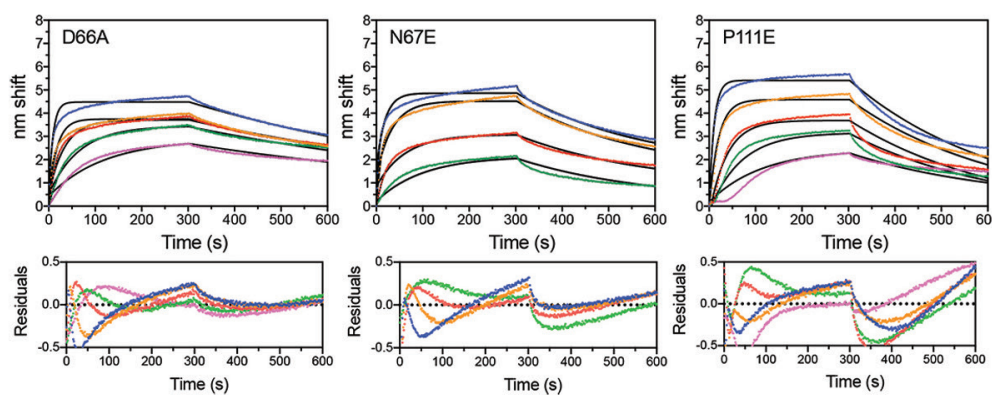

Fig. 3
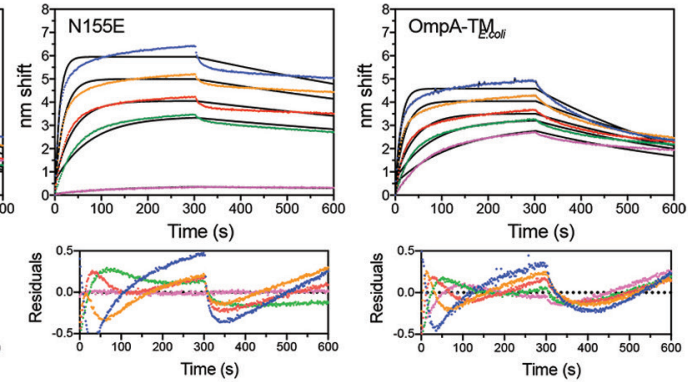

BLI sensorgrams are shown for crosslinked Sf6 and varying concentrations of various OmpA-TM analytes 62.5 (purple), 125 (green), 250 (red), 500 (orange), and 1,000 (blue) $\mathrm{nM}$ at $37^{\circ} \mathrm{C}$

Reference subtracted raw data are shown as points. Association and dissociation times were 300 s. Residuals are shown below the sensorgrams and are less than $10 \%$ of the total signal. Kinetic and equilibrium dissociation constants generated from the sensorgrams are shown in Table 2 .

Table 2. Equilibrium dissociation constants for Sf6 and variant OmpA-TMs at $37^{\circ} \mathrm{C}$

\begin{tabular}{llll}
\hline Protein & $\boldsymbol{k}_{\text {on }}\left(\mathbf{M}^{-1} \mathbf{s}^{-1}\right)$ & $\boldsymbol{k}_{\text {off }}\left(\mathbf{s}^{-1}\right)$ & $\boldsymbol{K}_{\mathrm{D}}(\mathbf{n M})$ \\
\hline D66A & $1.1 \times 10^{5}$ & $1.4 \times 10^{-3}$ & 12.9 \\
N67E & $5.2 \times 10^{4}$ & $3.4 \times 10^{-3}$ & 65.4 \\
P111E & $9.3 \times 10^{5}$ & $3.1 \times 10^{-3}$ & 32.8 \\
N155E & $9.9 \times 10^{5}$ & $6.8 \times 10^{-4}$ & 6.9 \\
OmpA-TM & $8.7 \times 10^{4}$ & $2.2 \times 10^{-3}$ & 24.8 \\
\hline
\end{tabular}

from E. coli, "OmpA-TM $\mathrm{T}_{\text {.coli }}$ " and four that deviated by single amino acid substitutions from the native $S$. flexneri sequence (D66A, N67E, P111E, and N155E). These variants were chosen to represent a broad range of phenotypes (Porcek and Parent, 2015). Here, we measured the kinetics of Sf6 binding to these various receptor types using BLI and calculated the kinetic and affinity constants for each (Fig. 3, and Table 2). Again, some complexity was evident but a simple 1:1 binding model fit the data better than two-state parallel or conformational change models. The only exception was the lowest analyte concentration for N67E (Fig. 3, magenta line), for which global fits did not converge, and was therefore eliminated from the analysis. The calculated parameters were consistent with fast-on and slow-off kinetics. OmpA-TM E.coli and all S. flexneri OmpA-TM variants bound Sf6 with $\mathrm{nM}$ affinity; the $\mathrm{K}_{\mathrm{D}} \mathrm{s}$ ranged between 6.9 and $65.4 \mathrm{nM}$. The kinetic parameters of binding for the variants differed only slightly when compared to those of

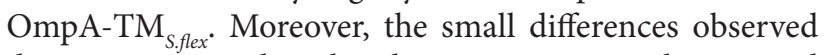
do not correspond to the phenotypes previously reported (Porcek and Parent, 2015). For example, Sf6 infection in Shigella cells expressing E. coli OmpA is ten-fold lower than on cells expressing $S$. flexneri OmpA. OmpA-TM E.coli $_{\text {in }}$ inable to efficiently induce genome ejection of Sf6 in vitro (Porcek and Parent, 2015), yet the binding affinities of Sf6 to OmpA-TM $\mathrm{T}_{\text {.coli }}$ and OMpAT-TM ${ }_{\text {S.flex }}$ are highly similar, 25 and 36 nM, respectively. Furthermore, N155E exhibited similar characteristics to E. coli OmpA yet displayed the highest affinity $(7 \mathrm{nM})$. Given the small range of affinities observed in the BLI data collected and the lack of correlation to our previous work, we interpret these kinetic differences as not significant, particularly given the experimental and instrumental set-up in BLI (Yang et al., 2016). Overall these data show that there are no large kinetic differences in the rates, at which Sf6 binds various forms of OmpA.

\section{Discussion}

To test our hypothesis that phenotypic differences in infection efficiencies of Sf6 on S. flexneri expressing variant OmpAs (Porcek and Parent, 2015) may be due to differences in binding affinities of Sf6 to OmpA, we purified six versions of OmpA-TM (S. flexneri, E. coli, and single amino acid substitutions in OmpA-TM S.flex $_{\text {D }}$ D6A, N67E, P111E, and $\mathrm{N} 155 \mathrm{E}$ ). To determine the kinetic parameters of Sf6 and OmpA-TMs, whole Sf6 virions were crosslinked to AR2G sensors and changes in the interference of white light using BLI to generate sensorgrams were measured. Consistent with BLI and SPR studies published with purified phage proteins and host cells (Marti et al., 2013) or purified receptor proteins (Legrand et al., 2016), we determined the binding affinity of Sf6 to OmpA-TM S.flex $_{\text {o be }}$ to in affinity. Kinetics were faston and slow-off and fit a simple one-state model reasonably well. Furthermore, OmpA-TM ${ }_{\text {E.coli }}$ and S. flexneri OmpA-TM variants bound Sf6 with similar affinities and their calculated kinetic parameters varied only slightly when compared to OmpA-TM S.flex $_{\text {. }}$

Previously published kinetic analyses performed with BLI for animal viruses and their respective host receptors have shown that binding affinities are in the $\mu \mathrm{M}-\mathrm{pM}$ 
range (Xiong et al., 2013; Yang et al., 2015; Zhang et al., 2015). Surface plasmon resonance (SPR), another optical biosensing technique, has also been used to study virus:host interactions. For example, Bonaparte et al. showed that the equilibrium dissociation constant for Hendra virus attachment glycoprotein to its receptor, human ephrin-B2 is $1 \mathrm{nM}$ (Bonaparte et al., 2005). Another SPR study showed that purified receptor binding proteins of human coronavirus, Middle East respiratory syndrome (MERS-CoV), and a bat coronavirus HKU4 can bind to human CD26 with $\mathrm{K}_{\mathrm{D}} \mathrm{s}$ of $18.4 \mathrm{nM}$ and $35.7 \mu \mathrm{M}$, respectively (Wang et al., 2014). Recently, Marti et al. showed via SPR that the binding affinity of the long tail fiber of bacteriophage S16, the phage tail protein that mediates interaction with the host, and its host Salmonella enterica ssp. enterica is $\sim 5 \mathrm{nM}$ (Marti et al., 2013).

The data presented herein suggest that the previously reported differences in Sf6 infection efficiencies seen in vivo and the differences in Sf6 genome ejection efficiencies in vitro (Porcek and Parent, 2015) are not based solely on the kinetics of receptor binding. There are no significant kinetic differences between Sf6 binding to the various OmpA-TMs, nor are there any significant changes when temperature is varied, even though both factors have been shown to cause changes in infection efficiency. Our results are similar to those in another study, in which the authors use SPR and demonstrate that amino acid substitutions in the coronavirus receptor binding protein do not greatly affect the overall kinetic parameters when binding to the human receptor CD26 (Wang et al., 2014). Their data support the idea that virus: host recognition is more complex and not dependent solely upon binding affinities. Our work, in combination with the coronavirus data, suggest that this may be a common theme throughout virology, and a phenomenon that may be universally conserved across kingdoms from bacteriophage to eukaryotic viruses. Kinetics alone do not explain the changes in infection efficiencies observed when temperature is varied or when amino acid substitutions are present in the receptor protein. Therefore, we hypothesize that conformational changes in the phage upon interaction with receptors are key to effective host recognition.

The current working model for Podoviridae attachment is a three step model (Casjens and Molineux, 2012). In the first step the virion binds to LPS reversibly. In the second step there is likely an irreversible interaction with a secondary receptor. Third, the genome is translocated from the phage capsid into the cell concurrently with several conformational changes in virion structure. Hu et al. have shown that bacteriophage T7 (Hu et al., 2013) undergoes extensive structural remodeling during infection, particularly in the tail machinery. In summation, we propose a model in which Sf6: Omp receptor recognition is not solely based on kinetics, but likely also involves conformational changes induced when docking to a cell surface (Fig. 4). Sf6 interacts with LPS first

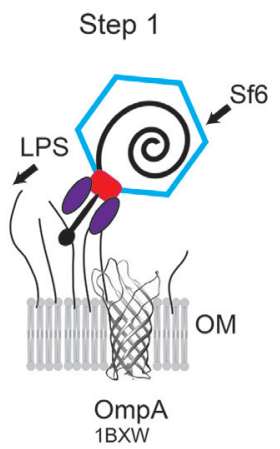

Step 2

Step 3
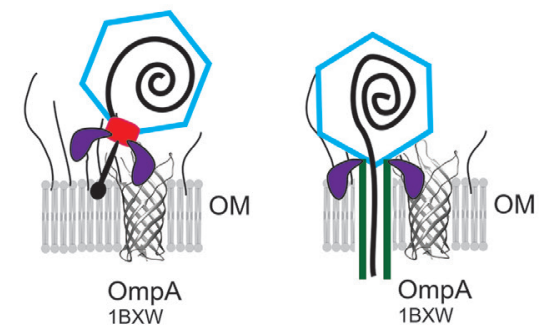

Fig. 4

Schematic showing steps in Sf6 attachment (modified from (Casjens and Molineux, 2012))

Step 1: A virion, likely coming in at an angle, binds to lipopolysaccharide (LPS). Step 2: The tailspike proteins (purple) hydrolyze the LPS, bringing the virion closer to the outer membrane (OM) surface, where it then interacts with OmpA. The crystal structure of E. coli OmpA (PDB: 1BXW) is depicted as a ribbon diagram using UCSF Chimera (Pettersen et al., 2004). Interaction with OmpA likely triggers a conformational change in the tail machinery. Step 3: dsDNA likely enters the cell through a channel formed by the tail and the ejection proteins. Schematic is not to scale.

via its tailspikes (Muller et al., 2008; Casjens and Molineux, 2012; Parent et al., 2014), likely coming into contact with the host surface at an angle, as work with a closely related phage, P22, has shown (Wang et al., 2017). Once Sf6 has cleaved enough LPS repeats (Freiberg et al., 2003; Andres et al., 2010) and is close enough to the surface of the cell, it interacts with its secondary receptor, an Omp (Parent et al., 2014). Upon interaction with Omps by the tail machinery, a conformational change in the phage is likely triggered. Amino acid substitutions in the loops of OmpA may affect the ability of the phage to adopt the correct conformation to promote channel formation, which is necessary to translocate the DNA genome (Casjens and Molineux, 2012; Bhardwaj et al., 2014, 2016; Wu et al., 2016). Although more work is necessary to discern a complete understanding of Sf6 (and Podoviridae) infection, the data presented here shed light on the kinetics of Sf6 and OmpA binding, which is an important step during host recognition. All of the work presented here was completed solely with purified OmpA. Our previous work showed that keeping LPS constant, but varying different OmpAs resulted in differences of ejection efficiencies and rates in vitro (Porcek and Parent, 2015). Since both components are required for infection (Parent et al., 2014; Porcek and Parent, 2015), it may be possible that the LPS helps to "prime" the phage to interact with OmpA, by inducing a subtle, initial conformational change that could induce different interactions with OmpA loop variants. Future work includes building on our current platform and will include a much more complex binding landscape. Ultimately, we 
hope to expand these studies to liposomes containing LPS and OmpA and/or whole $S$. flexneri cells to better elucidate the mechanistic properties of the Sf6 infection process.

Acknowledgments. The authors would like to thank Dr. Kristin Parent (MSU) for collaborative assistance, use of her laboratory and helpful comments on the manuscript, Dr. Xuefei Huang (MSU) and Peng Wang for time on their BLI instrument and Dr. Charles Hoogstraten and Senem Aykul for thoughtful discussion and help with kinetic analysis.

\section{References}

Abdiche Y, Malashock D, Pinkerton A, Pons J (2008): Determining kinetics and affinities of protein interactions using a parallel real-time label-free biosensor, the Octet. Analyt. Biochem. 377, 209-217. https://doi.org/10.1016/j. ab.2008.03.035

Andres D, Hanke C, Baxa U, Seul A Barbirz S, Seckler R (2010): Tailspike interactions with lipopolysaccharide effect DNA ejection from phage P22 particles in vitro. J. Biol. Chem. 285, 36768-36775. https://doi.org/10.1074/jbc. $\underline{\text { M110.169003 }}$

Andres D, Roske Y, Doering C, Heinemann U, Seckler R, Barbirz $S$ (2012): Tail morphology controls DNA release in two Salmonella phages with one lipopolysaccharide receptor recognition system. Mol. Microbiol. 83, 1244-1253. https://doi.org/10.1111/j.1365-2958.2012.08006.x

Baptista C, Santos MA, Sao-Jose C (2008): Phage SPP1 reversible adsorption to Bacillus subtilis cell wall teichoic acids accelerates virus recognition of membrane receptor YueB. J. Bacteriol. 190, 4989-4996. https://doi.org/10.1128/ JB.00349-08

Baxa U, Steinbacher S, Miller S, Weintraub A, Huber R, Seckler R (1996): Interactions of phage P22 tails with their cellular receptor, Salmonella O-antigen polysaccharide. Biophys. J. 71, 2040-2048. https://doi.org/10.1016/S00063495(96)79402-X

Bertozzi Silva J, Storms Z, Sauvageau D (2016): Host receptors for bacteriophage adsorption. FEMS Microbiol. Lett. 363, pii: fnw002. https://doi.org/10.1093/femsle/fnw002

Bhardwaj A, Olia AS, Cingolani G (2014): Architecture of viral genome-delivery molecular machines. Curr. Opin. Struct. Biol. 25, 1-8. https://doi.org/10.1016/j.sbi.2013.10.005

Bhardwaj A, Sankhala RS, Olia AS, Brooke D, Casjens SR, Taylor DJ, Prevelige PE, Jr., Cingolani G (2016): Structural Plasticity of the Protein Plug That Traps Newly Packaged Genomes in Podoviridae Virions. J. Biol. Chem. 291, 215-226. https://doi.org/10.1074/jbc.M115.696260

Bonaparte MI, Dimitrov AS, Bossart KN, Crameri G, Mungall BA, Bishop KA, Choudhry V, Dimitrov DS, Wang LF, Eaton BT, Broder CC (2005): Ephrin-B2 ligand is a functional receptor for Hendra virus and Nipah virus. Proc. Natl. Acad. Sci. USA 102, 10652-10657. https:// doi.org/10.1073/pnas.0504887102
Braun V, Wolff H (1973): Characterization of the receptor protein for phage $\mathrm{T} 5$ and colicin $\mathrm{M}$ in the outer membrane of $\mathrm{E}$. coli B. FEBS Lett. 34, 77-80. https://doi.org/10.1016/00145793(73)80707-0

Burguillo FJ, Wright AJ, Bardsley WG (1983): Use of the F test for determining the degree of enzyme-kinetic and ligandbinding data. A Monte Carlo simulation study. Biochem. J. 211, 23-34. https://doi.org/10.1042/bj2110023

Casjens, S, Winn-Stapley DA, Gilcrease EB, Morona R, Kuhlewein C, Chua JE, Manning PA, Inwood W, Clark AJ (2004): The chromosome of Shigella flexneri bacteriophage Sf6: complete nucleotide sequence, genetic mosaicism, and DNA packaging. J. Mol. Biol. 339, 379-394. https://doi. org/10.1016/j.jmb.2004.03.068

Casjens, SR, and Molineux, IJ (2012): Short noncontractile tail machines: adsorption and DNA delivery by podoviruses: Adv Exp Med Biol, Vol. 726. pp. 143-179. https://doi. org/10.1007/978-1-4614-0980-9 7

Chatterjee, A (1969): Use of bacteriophage-resistant mutants to study the nature of the bacteriophage receptor site of Staphylococcus aureus. J. Bacteriol. 98, 519-527.

Cleary PP, Wannamaker LW, Fisher M, Laible N (1977): Studies of the receptor for phage A25 in group A streptococci: the role of peptidoglycan in reversible adsorption. J. Exp. Med. 145, 578-593. https://doi.org/10.1084/ jem.145.3.578

Concepcion J, Witte K, Wartchow C, Choo S, Yao D, Persson H, Wei J, Li P, Heidecker B, Ma W, Varma R, Zhao LS, Perillat D, Carricato G, Recknor M, Du K, Ho H, Ellis T, Gamez J, Howes M, Phi-Wilson J, Lockard S, Zuk R, Tan H (2009): Label-free detection of biomolecular interactions using BioLayer interferometry for kinetic characterization. Combinatorial Chemistry \& High Throughput Screening 8, 791-800. https://doi.org/10.2174/138620709789104915

de Frutos M, Letellier L, Raspaud E (2005): DNA ejection from bacteriophage T5: analysis of the kinetics and energetics. Biophys. J. 88, 1364-1370. https://doi.org/10.1529/ biophysj.104.048785

Drexler K, Riede I, Montag D, Eschbach M, Henning U (1989): Receptor Specificity of the Escherichia coli T-even type phage Ox2. J. Mol. Biol. 207, 797-803. https://doi. org/10.1016/0022-2836(89)90245-3

Flint SJ, Enquiest LW, Krug RM, Racaniello VR, Skalka AM (2000): Principles of Virology, Molecular biology, Pathogenesis, and Control. ASM Press.

Freiberg A, Morona R, Van den Bosch L, Jung C, Behlke J, Carlin N, Seckler R, Baxa U (2003): The tailspike protein of Shigella phage Sf6. A structural homolog of Salmonella phage P22 tailspike protein without sequence similarity in the beta-helix domain. J. Biol. Chem. 278, 1542-1548. https://doi.org/10.1074/jbc.M205294200

Gonzalez-Garcia VA, Pulido-Cid M, Garcia-Doval C, Bocanegra R, van Raaij MJ, Martin-Benito J, Cuervo A, Carrascosa JL (2015): Conformational changes leading to T7 DNA delivery upon interaction with the bacterial receptor. J. Biol. Chem. 290, 10038-10044. https://doi.org/10.1074/ jbc.M114.614222 
Hantke K, Braun V (1978): Functional interaction of the tonA/ tonB receptor system in Escherichia coli. J. Bacteriol. 135, 190-197.

Hashemolhosseini S, Holmes Z, Mutschler B, Henning U (1994a): Alterations of receptor specificities of coliphages of the T2 family. J. Mol. Biol. 240, 105-110. https://doi.org/10.1006/ ¡mbi.1994.1424

Hashemolhosseini S, Montag D, Kramer L, Henning U (1994b): Determinants of receptor specificity of coliphages of the T4 family. A chaperone alters the host range. J. Mol. Biol. 241, 524-533. https://doi.org/10.1006/jmbi.1994.1529

Hendrix RW (2002): Bacteriophages: evolution of the majority. Theor Popul Biol 61, 471-80. https://doi.org/10.1006/ tpbi.2002.1590

Hu B, Margolin W, Molineux IJ, Liu J (2013): The bacteriophage T7 virion undergoes extensive structural remodeling during infection. Science 339, 576-579. https://doi.org/10.1126/ science. 1231887

Killmann H, Videnov G, Jung G, Schwarz H, Braun V (1995): Identification of receptor binding sites by competitive peptide mapping: phages T1, T5, and phi 80 and colicin $\mathrm{M}$ bind to the gating loop of FhuA. J. Bacteriol. 177, 694-698. https://doi.org/10.1128/jb.177.3.694-698.1995

Kleinschmidt JH, Wiener MC, Tamm LK (1999): Outer membrane protein A of E. coli folds into detergent micelles, but not in the presence of monomeric detergent. Protein Sci. 8, 2065-2071. https://doi.org/10.1110/ps.8.10.2065

Legrand P, Collins B, Blangy S, Murphy J, Spinelli S, Gutierrez C, Richet N, Kellenberger C, Desmyter A, Mahony J, van Sinderen D, Cambillau C (2016): The atomic structure of the phage Tuc2009 baseplate tripod suggests that host recognition involves two different carbohydrate binding modules. MBio 7, e01781-15. https://doi.org/10.1128/ mBio.01781-15

Lindberg AA, Wollin R, Gemski P, Wohlhieter JA (1978): Interaction between bacteriophage Sf6 and Shigella flexneri. J. Virol .27, 38-44.

Mackay DJ, Bode VC (1976): Events in lambda injection between phage adsorption and DNA entry. Virology 72, 154-166. https://doi.org/10.1016/0042-6822(76)90320-2

Marti R, Zurfluh K, Hagens S, Pianezzi J, Klumpp J, Loessner MJ (2013): Long tail fibres of the novel broad-host-range T-even bacteriophage S16 specifically recognize Salmonella OmpC. Mol. Microbiol. 87, 818-834. https://doi. org/10.1111/mmi.12134

Morona R, Henning U (1984): Host range mutants of bacteriophage Ox2 can use two different outer membrane proteins of Escherichia coli K-12 as receptors. J. Bacteriol. 159, 579-582.

Morona R, Kramer C, Henning U (1985): Bacteriophage receptor area of outer membrane protein OmpA of Escherichia coli K-12. J Bacteriol 164, 539-543.

Morona R, Mavris M, Fallarino A, Manning PA (1994): Characterization of the rfc region of Shigella flexneri. J. Bacteriol. 176, 733-747. https://doi.org/10.1128/jb.176.3.733747.1994

Muller JJ, Barbirz S, Heinle K, Freiberg A, Seckler R, Heinemann $U$ (2008): An intersubunit active site between supercoiled parallel beta helices in the trimeric tailspike endorham- nosidase of Shigella flexneri Phage Sf6. Structure 16, 766-775. https://doi.org/10.1016/j.str.2008.01.019

Parent KN, Erb ML, Cardone G, Nguyen K, Gilcrease EB, Porcek NB, Pogliano J, Baker TS, Casjens SR (2014): OmpA and OmpC are critical host factors for bacteriophage Sf6 entry in Shigella. Mol. Microbiol. 92, 47-60. https://doi. org/10.1111/mmi.12536

Pettersen EF, Goddard TD, Huang CC, Couch GS, Greenblatt DM, Meng EC, Ferrin TE (2004): UCSF Chimera - a visualization system for exploratory research and analysis. J. Comput. Chem. 25, 1605-1612. https://doi.org/10.1002/ jcc. 20084

Poranen MM, Daugelavicius R, Bamford DH (2002): Common principles in viral entry. Annu. Rev. Microbiol. 56, 521-538. https://doi.org/10.1146/annurev.micro.56.012302.160643

Porcek NB, Parent KN (2015): Key Residues of S. flexneri OmpA Mediate Infection by Bacteriophage Sf6. J. Mol. Biol. 427, 1964-1976. https://doi.org/10.1016/j.jmb.2015.03.012

Rakhuba DV, Kolomiets EI, Dey ES, Novik GI (2010): Bacteriophage receptors, mechanisms of phage adsorption and penetration into host cell. Pol. J. Microbiol. 59, 145-155.

Randall-Hazelbauer L, Schwartz M (1973): Isolation of the bacteriophage Lambda receptor from Escherichia coli. J. Bacteriol. 116, 1436-1446.

Sao-Jose C, Lhuillier S, Lurz R, Melki R, Lepault J, Santos MA, Tavares P (2006): The ectodomain of the viral receptor YueB forms a fiber that triggers ejection of bacteriophage SPP1 DNA. J. Biol. Chem. 281, 11464-1170. https://doi. org/10.1074/jbc.M513625200

Smith SG, Mahon V, Lambert MA, Fagan RP (2007): A molecular Swiss army knife: OmpA structure, function and expression. FEMS Microbiol. Lett. 273, 1-11. https://doi. org/10.1111/j.1574-6968.2007.00778.x

Wang C, Tu J, Hu B, Molineux I, Liu J (2017): Visualizing Infection Initiation of Bacteriophage P22 by Cryo-Electron Tomography. Biophysical Journal. https://doi.org/10.1016/j. bpj.2016.11.1704

Wang Q, Qi J, Yuan Y, Xuan Y, Han P, Wan Y, Ji W, Li Y, Wu Y, Wang J, Iwamoto A, Woo PC, Yuen KY, Yan J, Lu G, Gao GF (2014): Bat origins of MERS-CoV supported by bat coronavirus HKU4 usage of human receptor CD26. Cell Host Microbe 16, 328-337. https://doi.org/10.1016/j. chom.2014.08.009

Wendlinger G, Loessner MJ, Scherer S (1996): Bacteriophage receptors on Listeria monocytogenes cells are the $\mathrm{N}$ acetylglucosamine and rhamnose substituents of teichoic acids or the peptidoglycan itself. Microbiology 142 ( Pt 4), 985-992. https://doi.org/10.1099/00221287-142-4-985 Wu W, Leavitt JC, Cheng N, Gilcrease EB, Motwani T, Teschke CM, Casjens SR, Steven AC (2016): Localization of the Houdinisome (Ejection Proteins) inside the Bacteriophage P22 Virion by Bubblegram Imaging. MBio 7. https://doi. org/10.1128/mBio.01152-16

Xiong X, Martin SR, Haire LF, Wharton SA, Daniels RS, Bennett MS, McCauley JW, Collins PJ, Walker PA, Skehel JJ, Gamblin SJ (2013): Receptor binding by an H7N9 influenza virus from humans. Nature 499, 496-49. https://doi. org/10.1038/nature12372 
Yang D, Singh A, Wu H, Kroe-Barrett R (2016): Comparison of biosensor platforms in the evaluation of high affinity antibody-antigen binding kinetics. Anal. Biochem. 508, 78-96. https://doi.org/10.1016/j.ab.2016.06.024

Yang H, Carney PJ, Chang JC, Guo Z, Villanueva JM, Stevens $J$ (2015): Structure and receptor binding preferences of recombinant human $\mathrm{A}(\mathrm{H} 3 \mathrm{~N} 2)$ virus hemagglutinins. Virology 477, 18-31. https://doi.org/10.1016/j. virol.2014.12.024
Young FE (1967): Requirement of glucosylated teichoic acid for adsorption of phage in Bacillus subtilis 168. Pro.c Natl. Acad. Sci. USA 58, 2377-2384. https://doi.org/10.1073/ pnas.58.6.2377

Zhang H, de Vries RP, Tzarum N, Zhu X, Yu W, McBride R, Paulson JC, Wilson IA (2015): A human-infecting H10N8 influenza virus retains a strong preference for avian-type receptors. Cell Host Microbe 17, 377-384. https://doi. org/10.1016/j.chom.2015.02.006 